\title{
CHARACTERIZATION OF ULTRAFAST InGaAs PHOTOCONDUCTORS AND THEIR APPLICATION TO SIGNAL PROCESSING IN RADIO-OVER-FIBRE TELECOMMUNICATIONS
}

\author{
R. Horvath, J.-F. Roux, J.-L. Coutaz, and J. Poëtte \\ Université Grenoble Alpes, Université Savoie Mont-Blanc, Grenoble INP, CNRS, IMEP-LAHC, 38000 Grenoble, France \\ Email: jean-francois.roux@univ-smb.fr
}

Received 1 February 2018; accepted 22 March 2018

\begin{abstract}
Characterization of nitrogen ion implanted InGaAs photoconductive devices is performed in both the electronic and optoelectronic regime. In the dark state, the optoelectronic device shows a dark resistance of a few $\mathrm{k} \Omega$ and a wide electrical bandwidth in the RF domain. This large bandwidth is also confirmed in the illuminated state, optoelectronic autocorrelation experiments performed with ultra-short optical pulses allow us to measure the picosecond optoelectronic time response of the device. In the second part of the work, we investigate the potentiality of this component to be used in the photonic assisted heterodyne detection of RF modulated carriers for radio-over-fibre telecommunications. We demonstrate that this photoconductor, thanks to its properties, can be used to demodulate complex data modulation formats in such an experiment.
\end{abstract}

Keywords: III-V material, high speed electronics, ion implanted InGaAs, metal-semiconductor-metal devices, optoelectronic autocorrelation, radio-over-fibre telecommunications, ultrafast photoconductor

PACS: 85.60.Bt, 85.60.Gz, 84.40.Ua

\section{Introduction}

Ultrafast photoconductive switches (PSWs), also known as Auston switches, are key components in high frequency optoelectronics [1, 2]. Indeed, they are not only used as photodetectors capable of characterizing fast optical transits, but they can also be used for numerous applications that involve optical generation of short electrical transients. For instance, when triggered by an ultrafast femtosecond laser, they can act as terahertz $(\mathrm{THz})$ sources and detectors for spectroscopy [3], generation of high amplitude electrical picosecond pulses [4], electrical samplers for photonic assisted analog-to-digital converters [5], or used to modulate RF signals [6]. In many applications, the required performances of the PSW are the response time in the picosecond scale, a high dark resistance, a high breakdown voltage and a good optical responsivity.
The ultrafast response time of such metal-semiconductor-metal (MSM) device can be obtained by reducing the photocarrier transit time down to a picosecond or less by shrinking the size of the electrode spacing to the nanometre scale [f]. Another approach is to limit the photocarrier lifetime of the semiconductor thanks to a dramatic increase in the concentration of carrier traps [ 8$]$. This latter approach gave rise to a very fruitful research topic in material science during the 1990-2000 years. Hence ultrafast non-stoichiometric III-V materials have been elaborated using low-temperature growth [9], ion implantation [10] or ion irradiation [11]. Consequently, low-temperature grown (LTG) GaAs, which is a material that matches almost all the requirements described above, can now be considered as a choice semiconductor of mature technology. For instance, LTG-GaAs PSW antennas associated 
with a pulsed laser operating in the $0.8-\mu \mathrm{m}$ region are at the heart of most of the commercial $\mathrm{THz}$ time-domain spectrometers.

For telecommunications wavelength around $1.55 \mu \mathrm{m}$, in addition to the applications described above, the research in high frequency optoelectronics is mostly driven by telecommunications and by the needs for new components that would be useful for very high data rate processing. Especially, radio-over-fibre (RoF) wireless communication with RF or sub-THz carrier frequency and multi Gbit/s optical and RF complex modulation formats is a rich area of research as it covers both high-speed electronics, telecommunications and $\mathrm{THz}$ physics [12, 13]. Ultrafast PSWs presenting a large electronic and optoelectronic bandwidth may find their place in such a technology. Actually, in these high bandwidth systems, one identified key function is the mixing of RF and optical signals. This requires that the mixing process offer a very high bandwidth together with linear responses to both the optical and electrical signals. Because of their intrinsic concept that makes them sensitive to both the optical and electrical signals, ultrafast PSWs can handle this function in a simple and cost effective manner. As a proof of concept, up- and down-conversions of $20 \mathrm{GHz}$ RF signal with an optical local oscillator (LO) consisting of a modulated $1.55 \mu \mathrm{m}$ laser have been demonstrated using an InGaAs based photoconductor 14. However, due to the long, nanosecond carrier lifetime of intrinsic InGaAs used in this work, the LO frequency was limited to $300 \mathrm{MHz}$, making this component not suitable for high data rate communication.

In the present paper, we investigate the use of an ultrafast InGaAs based PSW as an optoelectronic mixer. In the first part, we describe the component and fully characterize its optoelectronic performances including its ultrafast 1.2 ps response time. We then demonstrate its ability to mix optical and RF signals up to $67 \mathrm{GHz}$ and we conclude by proving its possible use in a data transmission RoF experiment.

\section{Device description}

In order to fabricate ultrafast PSW compatible with telecommunication wavelengths, research teams have adapted to InGaAs most of the techniques previously studied to grow ultrafast GaAs: low temperature growth [15], ion irradiation [16], ion implantation [17], or iron ( $\mathrm{Fe})$ doping [18] have been reported. Unfortunately, the picture is more contrasted with InGaAs because the obtained layers usually suffer from a low dark resistance. As a consequence, an additional technological process such as a hetero-structure design or mesaetching is required to obtain photoconductors with a reasonable low dark leakage current [19].

The device studied in this paper has been described in detail and partly characterized in [20]. Briefly, it is based on a $300-\mathrm{nm}$ thick InGaAs $25 \times 25 \mu \mathrm{m}^{2}$ mesa matched on a Fe:InP substrate. The layer has been irradiated with nitrogen atoms (2 $\mathrm{MeV}, 10^{13} \mathrm{~cm}^{-2}$ fluence) which create a large number of defects in the InGaAs layer and then penetrate in the Fe:InP substrate. Considering the implantation parameters, the concentration of EL2 type defects is estimated to be around $5 \times 10^{17} \mathrm{~cm}^{-3}$. The photoconductor gold electrodes are designed to act as a coplanar waveguide (CPW), the central line (width $25 \mu \mathrm{m}$ ) of which is interrupted at the mesa location by a $25 \mu \mathrm{m}$ gap with inter-digitated fingers as shown in the inset of Fig. 3. The number of fingers may be different (3 to 5 ) and their separation is consequently varying from 3 to $5 \mu \mathrm{m}$. The overall structure is about $750 \mu \mathrm{m}$ long and is ending with electrical pads that allow direct "on wafer" measurement using RF electrical coplanar probes.

\section{Device characterization}

As mentioned above, the DC electrical characteristics of the device have already been measured in [20]. We have checked that most of our samples have similar characteristics and confirmed the ohmic behaviour of the contacts. We then turn to the RF characterization of the device by measuring its $S$ (scattering) parameters in the dark state using a $0.04-65 \mathrm{GHz}$ vector network analyzer (VNA). The experimental frequency evolution of the transmission coefficient $\left(S_{21}\right.$ parameter $)$ is plotted in Fig. 1 for an unbiased and non-illuminated PSW. As expected, the PSW acts as a highpass filter and we can model its behaviour thanks to a very simple lumped-element equivalent electrical circuit as shown in the inset of Fig. 1 [2]. The experimental data is fitted with the theoretical response taking into account the equivalent 


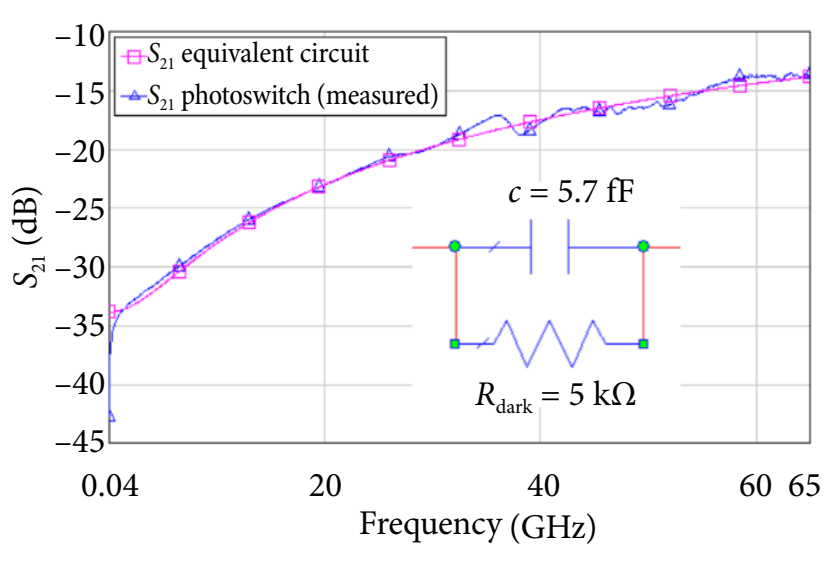

Fig. 1. $S_{21}$ parameter of the PSW in the dark state. The inset shows the lumped-element equivalent circuit of the device.

impedance of the coplanar waveguide $(50 \Omega)$. For the measured PSW, the best fit is obtained for an equivalent capacitance of $C=5.7 \mathrm{fF}$ and a dark resistance of $R_{\text {dark }}=5 \mathrm{k} \Omega$. Values in the same order of magnitude are found for the PSW design with other finger configurations.

So, considering the relative values of $C$ and $R_{\text {dark }}$, the behaviour of the device is mainly governed by the value of $R_{\text {dark }}$ for frequencies below $15 \mathrm{GHz}$, although we note that, close to DC, the experimental dark resistance seems to be larger than the value used for the modelling. Above $15 \mathrm{GHz}$, the impedance of the equivalent capacity $C$ becomes smaller than $2 \mathrm{k} \Omega$ and the response of the PSW to the RF signal is ruled by its feedthrough capacitance $C$. Consequently, in this frequency range, the value of the RF transmission $S_{21}$ does not depend on the value of $R_{\text {dark }}$ and it would have kept the same value even for larger values of $R_{\text {dark }}$. Finally, we conclude from this measurement that the low value of $C$ will also insure an ultrafast $R C$ response time (estimated to $300 \mathrm{fs}$ ) to femtosecond optical pulse excitation.

We then investigate the temporal response of the voltage-biased PSW to femtosecond optical pulses. When excited by an optical pulse with photon energy above the bandgap of the InGaAs mesa, the conductance of the PSW increases due to the photogeneration of free carriers in the absorbing layer. This reduces the electrical impedance of the PSW almost instantaneously and gives rise to an electrical pulse propagating in the PSW and the surrounding microwave waveguide. Generally, in PSWs, the temporal shape of the gener- ated pulses is governed by the carrier dynamics (including trapping, recombination and fast transit in the circuit) and the electrical $R C$ response. In the case of PSW made of ultrafast materials, the dominant process is the trapping of the photocarriers, which leads to the ps response time. Such a short pulse duration can hardly be determined by conventional oscilloscopes, which exhibit a limited response time, so alternative optoelectronic techniques, based on time-equivalent sampling, have been developed [2, 21, 22]. Unfortunately, these high-performance methods do not sense the electrical pulse at the PSW location but only after it has propagated along the CPW over few hundreds of $\mu \mathrm{m}$ or more. The electrical pulse shape is thus altered because of losses, radiation and dispersion of the electromagnetic waves in the waveguide [23]. Deconvolution of the disturbed pulse by reverse computation is possible but requires an additional simulation step and a precise knowledge of the waveguide [24]. In 1991, K. Grigoras, A. Krotkus and A. Deringas introduced a smart technique that gives a direct insight about the PSW response [25], which they have already used in these years to probe the ultrafast response of low-temperature grown InGaAs [26]. Actually, their method gets rid of the waveguide properties by directly probing the optoelectronic state of the PSW at the PSW location. It takes advantage of the non-linear electrical response of the PSW and its surrounding electrical circuit (DC bias source, waveguide and load charge) to optical power. Actually, the overall circuit acts as a voltage divider where the PSW is an opticallycontrolled resistance. The electrical field that accelerates the photo-carriers in the PSW is getting smaller as the photocurrent is increasing with optical power, leading to the overall sub-linear PSW response. The temporal dynamic of this non-linearity depends on both the dynamics of the photocarriers in the material and also on the variation of the accelerating electrical field in the structure. K. Grigoras et al. proposed to evaluate these dynamics by using two delayed optical pulses to excite the biased PSW, the first pulse being "perturbating" the PSW, whereas the second, delayed, pulse is sensing the dynamics of this perturbation. This experiment derived from the "pump-andprobe" technique, well-known in ultrafast optics, finds its temporal resolution in the shortness of 
the optical pulses, so it is easy to reach a sub-ps resolution with a femtosecond pulsed laser.

Here we perform such an experiment with a femtosecond fibre-laser operating at $1.55 \mu \mathrm{m}$ with a repetition rate of $100 \mathrm{MHz}$ (Fig. 2). Two delayed optical pulse trains are injected in one single optical fibre, which consists of 3 meters of a SMF28 fibre and a section of a dispersion compensated fibre (DCF). The length of the DCF is adjusted so that the optical pulses have the shortest duration at the PSW location. We checked that this duration is limited to less than 100 fs by measuring the optical autocorrelation trace thanks to the second harmonic generation in a $\mathrm{BBO}$ non-linear crystal (experiment not shown in Fig. 2). This optical autocorrelation trace is plotted in Fig. 3 (a continuous grey line): it also gives the zero delay time $(\Delta t=0)$ of the experimental set-up. Finally, we recorded the optoelectronic autocorrelation trace for a $2 \mathrm{~V}$ voltage bias and a $2.5 \mathrm{~mW}$ optical average power (Fig. 3, a dashed grey line). Apart from a very sharp peak around the zero delay, which is the signature of residual optical interferences be- tween the two optical pulses, this autocorrelation trace is well fitted with the double side exponential function: $A_{1} \exp \left(-t / \tau_{1}\right)+A_{2} \exp \left(-t / \tau_{2}\right)$. The best fit gives $A_{1}=0.6, A_{2}=0.2, \tau_{1}=1.2 \mathrm{ps}$ while $\tau_{2}=20 \mathrm{ps}$ (a black line). These ultrafast time constants $t_{1}$ and $t_{2}$ confirm the picosecond response time of these nitrogen-implanted InGaAs PSW.

Next we check the longer response of the PSW by measuring with a high bandwidth oscilloscope (Tektronic DSA 8200 with 80E10 sampling heads) the electrical pulses for different bias voltages. The data plotted in the inset of Fig. 4 indicates that the PSW response to $V_{\text {bias }}$ is linear, while the main plot clearly depicts the voltage pulse generated by the PSW. Its 27 ps FWHM duration is limited by the 15 ps rise time of the sampling head. At longer times, the signal is perturbed by fluctuations that are probably due to partial reflections of the pulses at the electrical probe location; we note that these reflections are at least one order of magnitude smaller than the main pulse generated by the biased illuminated PSW, confirming the ultrafast response of the latter.

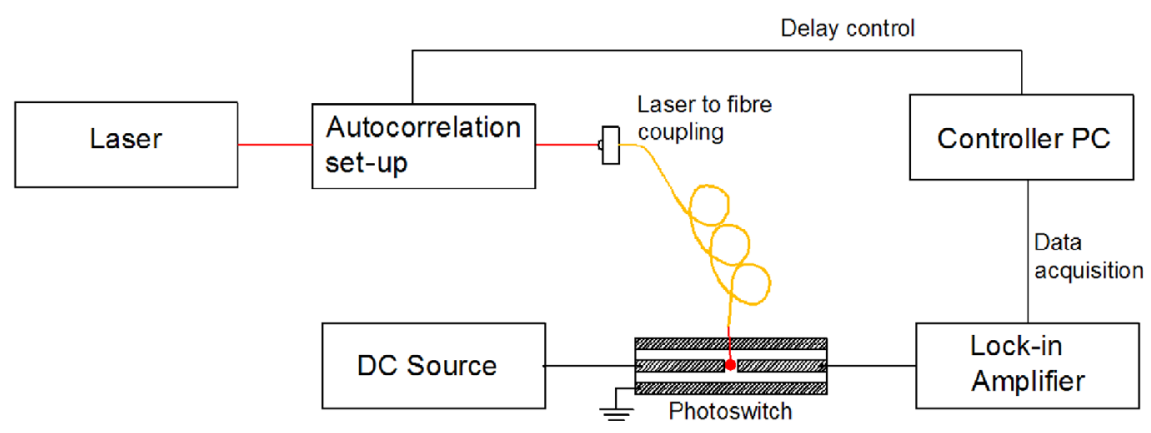

Fig. 2. Experimental setup to measure the optoelectronic response of the PSW. Autocorrelation set-up consists of an interferometer with a motor-controlled optical delay line.

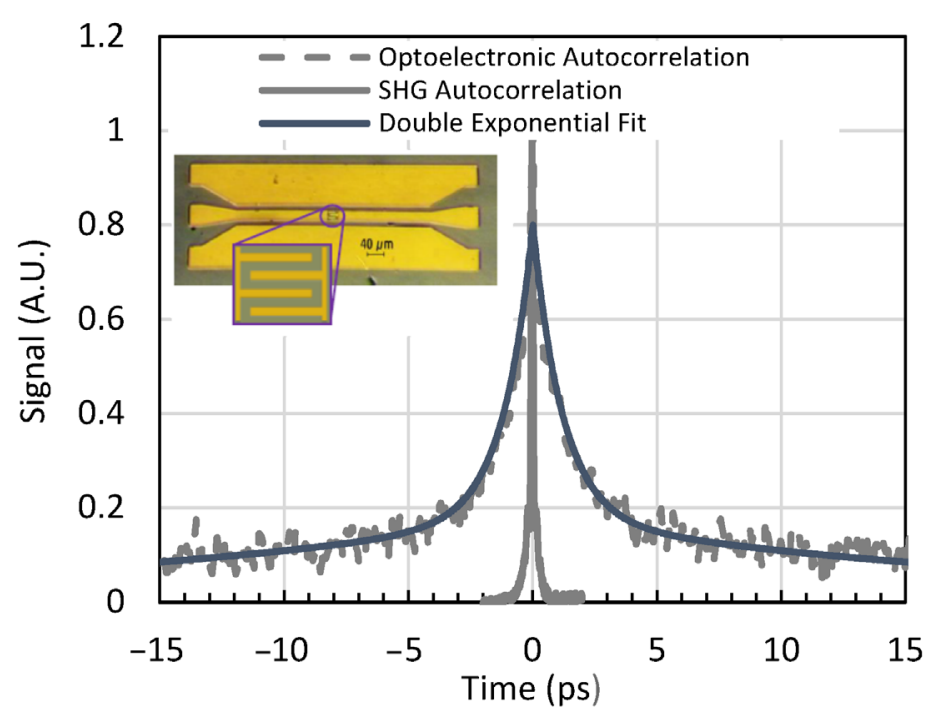

Fig. 3. Optoelectronic (a dashed grey line) and optical (grey line) autocorrelation traces of a nitrogen implanted InGaAs PSW (an inset). A double exponential fitting curve with time constants of 1.2 and 20 ps is also shown (a black line). 


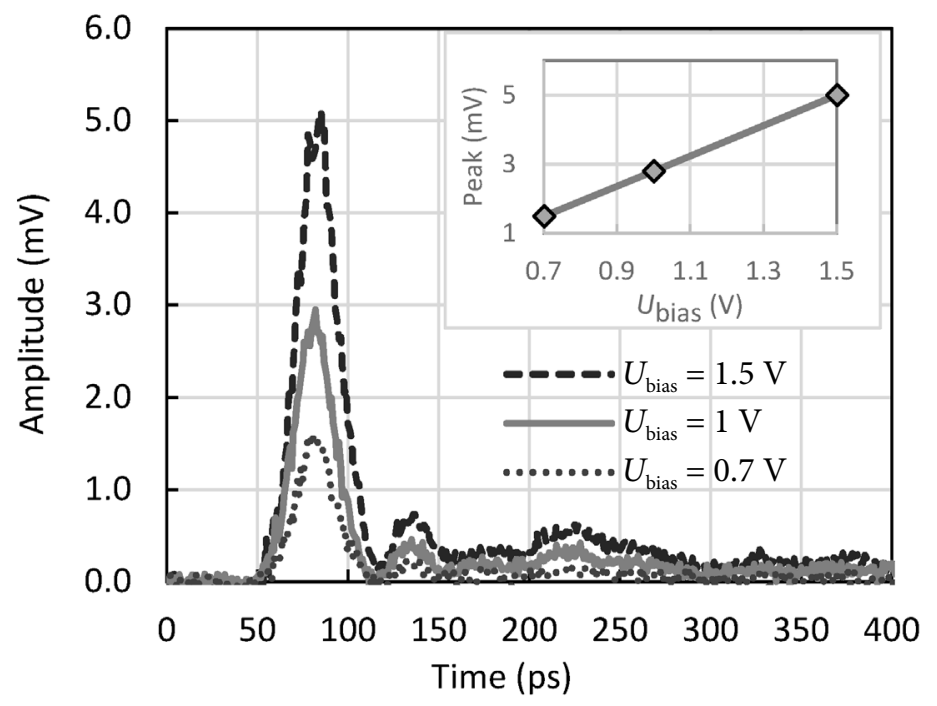

Fig. 4. Oscilloscope traces of the generated pulse for $5 \mathrm{~mW}$ average optical pump power and different bias voltages. The inset shows the voltage peak amplitude (in $\mathrm{mV}$ ) vs the bias voltage (in V).

\section{Heterodyne demodulation of amplitude modulated RF signals}

As mentioned above, one of the potential applications of ultrafast PSWs is modulation (or demodulation) of RF signals by optical means using the device as a hybrid optoelectronic mixer [ 6 ]. Such an application has already been investigated using phototransistors [27] or ultrafast unitravelling-carrier photodiodes (UTCPD) [28]. Phototransistors present low RF mixing losses but suffer from a limited bandwidth (few $\mathrm{GHz}$ ). Concerning the UTCPDs, they are more complex components to design; moreover, usually their optical-to-electrical conversion efficiency does not depend strongly on the electrical bias point so UTCPDs need to present a nonlinear electrical regime to be sensitive to the RF signal and used as a mixer [29]. On their side, MSM PSWs are simple and robust components, they exhibit a large bandwidth and their electrical output is intrinsically depending on both the optical and electrical (RF or DC) input signals.

Here we have investigated the use of the ultrafast InGaAs PSW to perform this operation in an experiment where the optical signal is a $1.55-\mu \mathrm{m} \mathrm{CW}$ beam modulated at the frequency $f_{\mathrm{LO}}$ and the DC biased voltage of the PSW is replaced by an RF input signal at the frequency $f_{\mathrm{RF}}$. Both optical and RF signals are mixed in the PSW because the photocurrent is the product of the photocarrier density (generated by the absorption of the $1.55 \mu \mathrm{m}$ optical beam) and the RF electrical field applied to the PSW, which accelerates the carriers. This mix- ing process results in an RF output signal oscillating at the intermediate frequency (IF) $f_{\mathrm{iF}}=f_{\mathrm{RF}}-f_{\mathrm{LO}}$. In our case, $f_{\mathrm{RF}}$ varies from 1 to $65 \mathrm{GHz}$, while the RF power ranges from -15 to $+15 \mathrm{dBm}$. As a modulated laser beam, we employ an InAs/InPbased quantum dash mode-locked laser diode self-oscillating at $58.625 \mathrm{GHz}$ with around $5 \mathrm{~mW}$ output power [30]. In order to compensate for the laser dispersion, we use $70 \mathrm{~m}$ of a SMF- 28 single mode fibre while a variable optical attenuator allows us to vary the average optical power that excites the PSW. The RF output of the PSW is detected thanks to a RF spectrum analyzer (RFSA) (Anritsu MS2668C $9 \mathrm{kHz}-40 \mathrm{GHz}$ ). For the RF input frequency in the range $19-67 \mathrm{GHz}, f_{\mathrm{iF}}$ falls within the allowed detection band of the RFSA. In Fig. 5, we plot the amplitude of this IF signal for an input RF power of $14 \mathrm{dBm}$. For an optical power of $5 \mathrm{~mW}$, the RF power at the IF is about $-65 \mathrm{dBm}$, leading to an estimated loss $P_{\mathrm{IF}} / P_{\mathrm{RF}}$ of $80 \mathrm{~dB}$. This loss is due to a low optical-to-electrical conversion, which is the drawback of the ultrafast response of our device.

We note that this large loss is within the same order of magnitude as the one presented by unbiased UTC photodiodes used as an optoelectronic mixer [29]. However, they might be strongly reduced by smart engineering of the photoconductive device [31]. Similarly to UTC photodiodes, the PSW presents an ultra large bandwidth of more than $65 \mathrm{GHz}$, as confirmed by its constant response over the whole frequency range, except in the vicinity of $58 \mathrm{GHz}$ where a systematic unexplained experimental decrease is observed. 


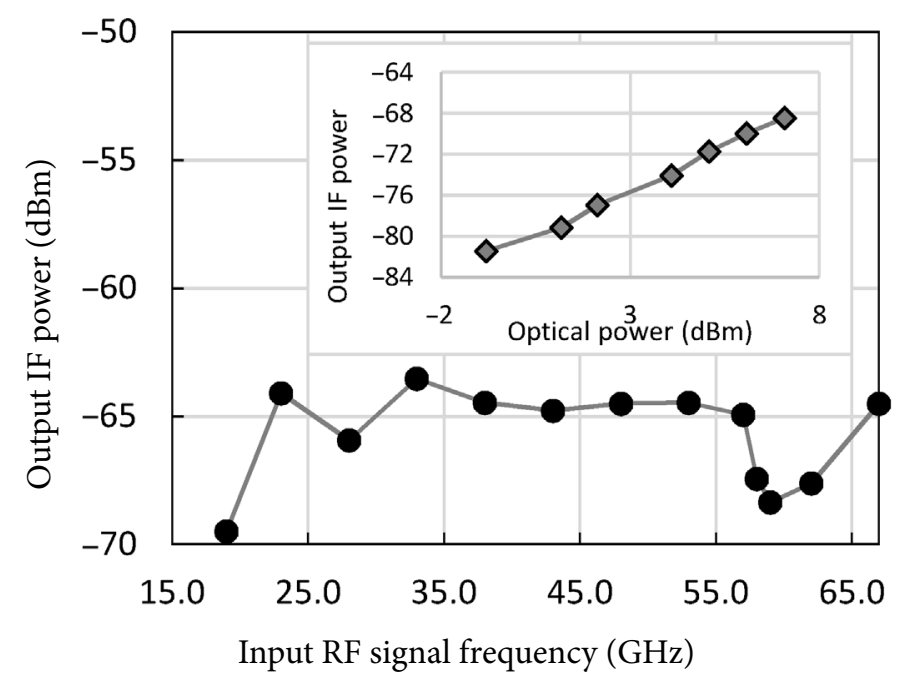

Fig. 5. Power of the IF signal for different input RF. The PSW is used as a heterodyne mixer in between a $14 \mathrm{dBm} \mathrm{RF}$ input signal and a $5 \mathrm{~mW}$ laser self-oscillating at $58.625 \mathrm{GHz}$. Inset: IF power dependence on the optical power for an RF of $57 \mathrm{GHz}$.
Finally, we have experimentally observed the linear response of the photomixer versus the RF input power (not shown here), whereas the power of the IF signal varies as the optical power to the power 1.6, demonstrating that the losses of the mixer may be reduced by increasing the impinging optical power (the inset of Fig. 5).

\section{Downconversion and demodulation of high- frequency data streams in a RoF experiment}

The hybrid mixing capabilities of the photoconductive switch have been presented in the previous paragraph. This experiment can be extended to the down-conversion of high frequency datastreams in a RoF experiment. In this case, one of the two RF signals (either at $f_{\mathrm{LO}}$ or $f_{\mathrm{RF}}$ ) is modulated to carry data as the other one will play the role of the local oscillator commonly used in a heterodyne detection experiment. So in the case of a usual RoF experiment, the optical signal is first detected by a photodiode which output is then mixed with the RF local oscillator in an RF mixer. Here we demonstrate that the PSW can play both roles (photodetector and mixer) simultaneously. For this demonstration, we replaced the MLL la- ser diode self-oscillating at $58.625 \mathrm{GHz}$ by a continuous wave DFB laser emitting at $1.55 \mu \mathrm{m}$ and we modulated it at the LO frequency $f_{\text {LO }}$ thanks to a Mach-Zehnder modulator (MZM) at its quadrature point. The RF mixed signal at the output of the PSW was analysed with a digital sampling oscilloscope (DSO) with a software capable of demodulating complex modulation formats. The setup configuration scheme is depicted in Fig. 6 .

In order to compensate the previously-measured $80 \mathrm{~dB}$ mixing losses of the PSW, we used a $40 \mathrm{~dB}$ gain RF amplifier before the DSO. Because of its limited bandwidth of $1 \mathrm{GHz}$, this amplifier restricts the mixing possibilities as the output IF should be in the $0-1 \mathrm{GHz}$ range. The data-stream was generated with an arbitrary waveform generator in the baseband which was modulating a carrier of frequency $f_{\mathrm{RF}}$. As a modulation format, we select the quadrature phase shift keying (QPSK), which is the basic IQ modulation with 2 bits/symbol, 4 states on a constellation diagram [32]. Here we choose $f_{\mathrm{LO}}=6.2 \mathrm{GHz}$ to modulate the $1.55 \mu \mathrm{m}$ $\mathrm{CW}$ laser and $f_{\mathrm{RF}}=6.8 \mathrm{GHz}$. The optical power after the MZM was $2.3 \mathrm{dBm}$. The QPSK modulated signal is thus carried by the $6.2 \mathrm{GHz} \mathrm{RF}$ signal, itself modulating the laser light, while the RF local

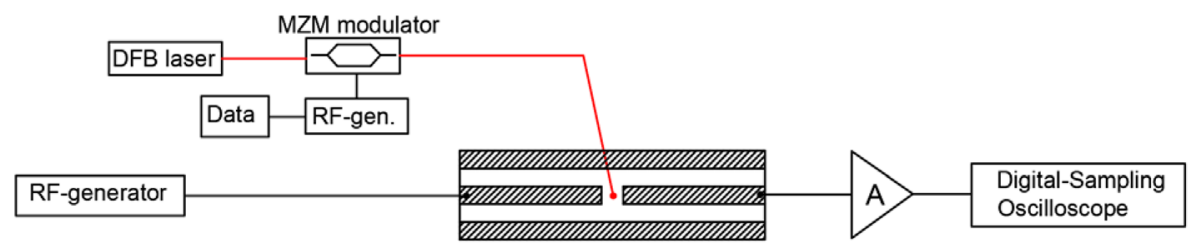

Fig. 6. Set-up of the data-stream demodulation experiment. 
oscillator is running at $6.8 \mathrm{GHz}$. We note that we have obtained similar results of demodulation by inverting the roles of the signals at $f_{\mathrm{LO}}$ and $f_{\mathrm{RF}}$, this latter being modulated by the QPSK modulation.

The flow of data was frequency down-converted to the IF frequency of $0.8 \mathrm{GHz}$ by the photoswitch under laser illumination. The DSO records the received IF signal and demodulates the received data-stream in real-time. As a figure of merit of the demodulated data-stream, we determine the error vector magnitude (EVM), calculated from the demodulated constellation of the received signal in the DSO. Table 1 shows typical EVM values for the experimental bit-rates, while a typical constellation diagram for the received signal is plotted in Fig. ㄱ.

Table 1. EVM results for different data rate. The value obtained for $150 \mathrm{Mbits} / \mathrm{s}$ was measured with an additional $3 \mathrm{~dB}$ of the input RF power.

\begin{tabular}{|c|c|}
\hline Data rate & EVM \\
\hline $50 \mathrm{Mbit} / \mathrm{s}$ & $11.78 \%$ \\
\hline $100 \mathrm{Mbit} / \mathrm{s}$ & $18.26 \%$ \\
\hline $150 \mathrm{Mbit} / \mathrm{s}$ & $22.38 \%$ \\
\hline
\end{tabular}

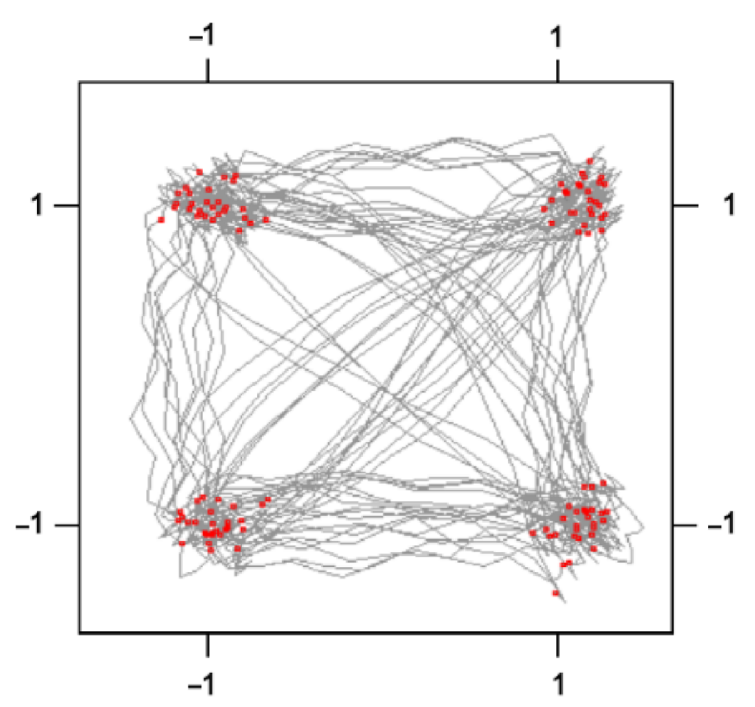

Fig. 7. Constellation diagram of the received $50 \mathrm{Mbit} / \mathrm{s}$ QPSK signal $(\mathrm{EVM}=11.78 \%)$.

These first experiments clearly demonstrate the functionality of the PSW as a mixer for RoF data demodulation. Here the performances in terms of bit-rate are limited both by our electronic instrumentation and by the RF losses induced by the PSW, which reduce the signal-to noise ratio of the modulated signal at IF. As already mentioned, an optimized design of the PSW will strongly reduce the RF mixing losses, while keeping the high bandwidth of the PSW.

\section{Conclusions}

In this work we have presented the first results of photoconductive mixing of optical and RF signals for QPSK data demodulation in a RoF experiment. We used nitrogen ion implanted InGaAs PSWs embedded in a coplanar waveguide to down-convert QPSK data carried by a $6.3 \mathrm{GHz} \mathrm{RF}$ signal. The PSW is characterized by $5 \mathrm{k} \Omega$ dark resistance and low equivalent capacitance that lead to a high electrical bandwidth. The optoelectronic response relaxation time of the PSW is about $1.2 \mathrm{ps}$, which results in a flat mixing efficiency from 15 up to $67 \mathrm{GHz}$. These preliminary results open the way to more advanced studies in RF wireless communications systems, in which high bit-rate data streams will be sampled and/or demodulated by optoelectronics means using such PSWs that are not only ultrafast but also simple, low-cost and robust.

\section{Acknowledgements}

We would like to thank Drs. C. Graham and C. Renaud from the Department of Electronic and Electrical Engineering, at the University College of London, UK, for providing us with the PSW. We are also grateful to Dr. F. Van Dijk from IIIV Labs, Palaiseau, France, who supplied us with the MLL diode.

This project has received funding from the European Union's Horizon 2020 Research and Innovation Program under the Marie SklodowskaCurie Grant Agreement No. 642355 FiWin5G.

\section{References}

[1] D.H. Auston, K.P. Cheung, and P.R. Smith, Picosecond photoconductive Hertzian dipoles, Appl. Phys. Lett. 45, 284-286 (1984).

[2] D.H. Auston, in: Picosecond Optoelectronic Devices, ed. C. H. Lee (Academic-Press, New York, 1983).

[3] D. Grischkowsky, S. Keiding, M. van Exter, and Ch. Fattinger, Far-infrared time-domain 
spectroscopy with terahertz beams of dielectrics and semiconductors, J. Opt. Soc. Am. B 7, 20062015 (1990).

[4] M.Y. Frankel, J.F. Whitaker, G.A. Mourou, F.W. Smith, and A.R. Calawa, High-voltage picosecond photoconductor switch based on lowtemperature-grown GaAs, IEEE Trans. Electron Devices 37, 2493-2498 (1990).

[5] L.Y. Nathawad, R. Urata, B. Wooley, and D.A.B. Miller, A 40-GHz-bandwidth, 4 bit, timeinterleaved $\mathrm{A} / \mathrm{D}$ converter using photoconductive sampling, IEEE J. Solid-State Circuits 38, 2021-2030 (2003).

[6] M.B. Kuppam, J.-F. Lampin, E. Peytavit, J.-F. Roux, and J.-L. Coutaz, Study of ultrafast semiconductor photoswitches for CW RF signal sampling and modulation, IEEE J. Lightwave Technol. 32, 3839-3845 (2014).

[7] S. Chou, Y. Liu, W. Khalil, H. Wan, T. Hsiang, and S. Alexandrou, Ultrafast nanoscale MSM photodetectors on bulk and low-temperature grown GaAs, Appl. Phys. Lett. 61, 819-821 (1992).

[8] A. Krotkus and J.-L. Coutaz, Non-stoichiometric semiconductor materials for terahertz optoelectronics applications, Semicond. Sci. Technol. 20, S142-S150 (2009).

[9] A. Krotkus, K. Bertulis, M. Kaminska, K. Korona, A. Wolos, J. Siegert, S. Marcinkevicius, J.-F. Roux, and J.-L. Coutaz, Be-doped low-temperaturegrown GaAs material for optoelectronic switches, IEE Proc. Optoelectron. 149, 111-115 (2002).

[10]A. Krotkus, S. Marcinkevicius, J. Jasinski, M. Kaminska, H.H. Tan, and C. Jagadish, Picosecond carrier lifetime in GaAs implanted with high doses of As ions: An alternative material to low-temperature GaAs for optoelectronic applications, Appl. Phys. Lett. 66, 3304-3306 (1995).

[11]A. Singh, S. Pal, H. Surdi, S.S. Prabhu, S. Mathimalar, V. Nanal, R.G. Pillay, and G.H. Döhler, Carbon irradiated semi insulating GaAs for photoconductive terahertz pulse detection, Opt. Exp. 23, 6656-6661 (2015).

[12]A.J. Seeds and K.J. Williams, Microwave photonics, J. Lightwave Technol. 24, 4628-4641 (2006).
[13]T. Nagatsuma, G. Ducournau, and C.C. Renaud, Advances in terahertz communications accelerated by photonics, Nat. Photonics 10, 371-379 (2016).

[14]H.-J. Song, T.-W. Kim, S.J. Jo, C.-H. Lim, K.-H. Oh, S.-G. Ihn, and J.-I. Song, Microwave photonic mixer utilizing an InGaAs photoconductor for radio over fibre applications, IEICE Trans. Electron. 90, 457-464 (2007).

[15]Y. Chen, S.S. Prabhu, S.E. Ralph, and D.T. McInturff, Trapping and recombination dynamics of low-temperature-grown InGaAs/ InAlAs multiple quantum wells, Appl. Phys. Lett. 72, 439-442 (1998).

[16]J. Mangeney, L. Joulaud, P. Crozat, and J.-M. Lourtioz, Ultrafast response ( $2.2 \mathrm{ps})$ of ion-irradiated InGaAs photoconductive switch at $1.55 \mu \mathrm{m}$, Appl. Phys. Lett. 83, 5551 (2004).

[17]C. Carmody, H.H. Tan, C. Jagadish, A. Gaarder, and S. Marcinkevicius, Ion-implanted $\mathrm{In}_{0.53} \mathrm{Ga}_{0.47}$ As for ultrafast optoelectronic applications, Appl. Phys. Lett. 82, 3913-3915 (2003).

[18]O. Hatem, J. Cunningham, E.H. Linfield, C.D. Wood, A.G. Davies, P.J. Cannard, M.J. Robertson, and D.G. Moodie, Terahertz-frequency photoconductive detectors fabricated from metal-organic chemical vapor deposition-grown Fe-doped InGaAs, Appl. Phys. Lett. 98, 121107 (2011).

[19]B. Globisch, R. Dietz, S. Nellen, T. Göbel, and M. Schell, Terahertz detectors from Be-doped lowtemperature grown InGaAs/InAlAs: Interplay of annealing and terahertz performance, AIP Adv. 6, 125011 (2016).

[20]C. Graham, R. Gwilliam, and A. Seeds, Nitrogen ion implanted InP based photo-switch, Opt. Exp. 20, 26696-26703 (2012).

[21]M. Bieler, H. Füser, and K. Pierz, Time-domain optoelectronic vector network analysis on coplanar waveguides, IEEE Trans. Microw. Theory Techn. 63, 3775-3784 (2015).

[22]H. Eusèbe, J.F. Roux, J.L. Coutaz, and A. Krotkus, Photoconductivity sampling of low-temperaturegrown Be-doped GaAs layers, J. Appl. Phys. 98, 033711 (2005). 
[23]M.Y. Frankel, S. Gupta, J.A. Valdmanis, and G.A. Mourou, Terahertz attenuation and dispersion characteristics of coplanar transmission lines, IEEE Trans. Microw. Theory Techn. 39, 910-916 (1991).

[24]C. Kastl, C. Karnetzky, A. Brenneis, F. Langrieger, and A. Holleitner, Topological insulators as ultrafast Auston switches in on-chip THz-circuits, IEEE J. Sel. Top. Quantum Electron. 23, 1-5 (2017).

[25]K. Grigoras, A. Krotkus, and A. Deringas, Picosecond lifetime measurement in semiconductor by optoelectronic autocorrelation, Electron. Lett. 27, 1024-1025 (1991).

[26] A. Krotkus, S. Marcinkevicius, K. Grigoras, V. Pasiskevicius, and J.A. Tellefsen, Ultrafast carrier relaxation in low-temperature grown $\operatorname{In}_{x} \mathrm{Ga}_{1-x}$ As layers, in: Proceedings of the 1994 Conference on Lasers and Electro-Optics Europe (Institute of Electrical \& Electronics Engineers (IEEE), USA, 1994) pp. 365-366.

[27]C.-S. Choi, H.-S. Kang, W.-Y. Choi, D.-H. Kim, and K.-S. Seo, Phototransistors based on InP
HEMTs and their applications to millimeter-wave radio-on-fibre systems, IEEE Trans. Microw. Theory Techn. 53, 256-263 (2005).

[28]A. Hirata, T. Furuta, H. Ito, and T. Nagatsuma, $10-\mathrm{Gb} / \mathrm{s}$ millimeter-wave signal generation using photodiode bias modulation, J. Lightwave Technol. 24, 1725-1731 (2006).

[29]E. Rouvalis, M. Fice, C. Renaud, and A. Seeds, Optoelectronic detection of millimetre-wave signals with travelling-wave uni-travelling carrier photodiodes, Opt. Express 19, 2079-2084 (2011).

[30]F. Brendel, J. Poëtte, B. Cabon, T. Zwick, F. van Dijk, F. Lelarge, and A. Accard, Chromatic dispersion in $60 \mathrm{GHz}$ radio-over-fibre networks based on mode-locked lasers, J. Lightwave Technol. 29, 3810-3816 (2011).

[31]E. Peytavit, F. Pavanello, G. Ducournau, and J.-F. Lampin, Highly efficient terahertz detection by optical mixing in a GaAs photoconductor, Appl. Phys. Lett. 103, 201107 (2013).

[32]B. Sklar, Digital Communication: Fundamentals and Applications, 2nd ed. (Prentice Hall, Upper Saddle River, 2009).

\title{
ULTRASPARČIŲJŲ InGaAs FOTOLAIDININKŲ CHARAKTERIZAVIMAS IR JŲ TAIKYMAS RADIJO ŠVIESOLAIDINĖS TELEKOMUNIKACIJOS SIGNALŲ APDOROJIMUI
}

\author{
R. Horvath, J.-F. Roux, J.-L. Coutaz, J. Poëtte \\ Grenoblio universitetas, Grenoblis, Prancüzija
}

